\title{
SHORT PULSE GENERATION IN SOLID STATE LASERS BY A NOVEL PASSIVE TECHNIQUE
}

\author{
M C MARCONI ${ }^{1}$, O E MARTINEZ ${ }^{2}$ and F P DIODATI \\ Cetlap (Citefa-Conicet) Zufriategu v Varela, 1603 Villa Martell, Argentına
}

Recelved 3 March 1987

\begin{abstract}
A novel passive Q-switching technique based on self focusing is studied theoretically and experimentally In this paper we present results obtained with a simple aberrationless gaussian model that describes the short pulse generation dynamics for different cavity configurations and different nonlınear characterıstıcs of the focusing medium Experımental results are also presented that are in good agreement with the predictions of the theoretıcal model Preliminary results in ultrashort pulse generation are also presented
\end{abstract}

\section{Introduction}

The passive generation of high power laser pulses has been an important research field in last years, and giant pulses obtained in solid state lasers have now many scientific and technical applications The most common technique employed to obtain passive gain modulation in a laser cavity makes use of the saturable absorption characteristic of some organic dyes Some devices like the Q-switch film introduced by KODAK represents a recent improvement in passive short pulse generation Nevertheless, the saturable absorption technique has the important drawback that the dye degrades principally from the UV radiation below $350 \mathrm{~nm}$ that breaks down the long chain dye molecules

The reliability of the system depends critically on the transmission coefficient of the absorber, and usually it is necessary to replace the dye volume (by circulating the dye in the cell) in order to assure the adequate emission of the laser Other disadvantages encountered in such systems were that a dye with an

Fellow from Consejo Nacional de Investıgaciones Cientificas y Tecnicas de la Republica Argentına Present address Eng1neerıng Research Center, Colorado State University, Fort Collins, CO 80523, U S A

2 Member of the Carrera del Investigador Cientifico del Consejo Nacional de Investigaciones Cientıficas y Tecnicas de la Republica Argentına absorbing peak in the laser wavelength must be used and that the bleaching process may favor the filament emission of the laser

In previous papers $[1,2]$, we described this new system that leads to a gain modulation and Q-switch emission The new technique makes use of the self focusing of the beam in a liquid with high nonlinear refractive index $n_{2}$ A cell with such liquid is placed in contact with one of the mirrors of an unstable cavity resonator When the intensity inside the cavity builds up, the self focusing reduces the diffraction losses by changing the effective radius of curvature of the mirror and creating a stable resonator configuration This causes the Q-switching of the cavity to occur

We present in this paper a complete theoretical and experimental study of this modulation technique Numerical simulation results using a simplified model are presented for different cavity configurations and for different values of the nonlinear refractive index of the focusing medium used as the passive modulation device We also present experimental results for the different conditions considered in the theoretical model While changing the temperature of the nonlinear medium and the cavity length, we controlled the second order refractive index and the initial cavity loss With these tests, we obtained experimental results that confirm the numerical pre- 


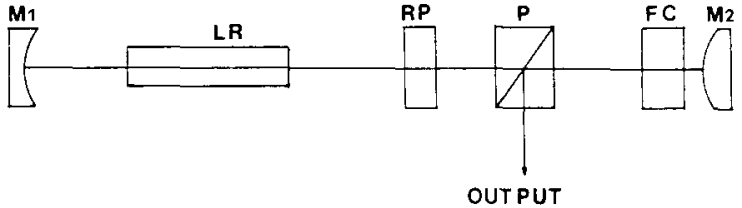

Fig 1 Experımental set-up Ml 100\% reflectıng mirror with radius of curvature $R_{1}=2 \mathrm{~m}$ M2 $100 \%$ reflectıng mirror with radius of curvature $R_{2}=-1 \mathrm{~m}$ contacted with the focusing, cell FC RP quarter wave plate $P$ polarizıng prism LR Nd glass laser rod

dictıons presented Prelıminary experımental results in ultrashort pulse generation are also presented

\section{Basic equations and numerical results}

Consider the cavity configuration shown in fig 1 The cavity length $L_{\mathrm{c}}$ was adjusted in order to obtain an unstable configuration In the gaussian mode approximation, the passive resonator can be described by a $2 \times 2$ matrix $A B C D$ [3-5] The matrix elements will be a function of the radius of curvature of the mirrors $\mathrm{R}_{1}$ and $\mathrm{R}_{2}$ and the cavity length $L_{c}$ If the reference plane is on the mirror $E_{1}$ with radius of curvature $R_{1}$, the matrix elements are

$A=1-L_{\mathrm{c}} / R_{2}, \quad B=2 L_{\mathrm{c}}\left(1-L_{\mathrm{c}} / R_{2}\right)$,

$C=\left(2 / R_{1} R_{2}\right)\left(2 L_{\mathrm{c}}-R_{1}-R_{2}\right)$,

$D=\left(1-2 L_{\mathrm{c}} / R_{2}-4 L_{\mathrm{c}} / R_{1}+4 L_{\mathrm{c}}{ }^{2} / R_{1} R_{2}\right)$

With this round trip magnification matrix $A B C D$, it 1s possible to obtain the resonator parameter $g=(A+D) / 2$ This definition of the resonator parameter $g$ leads to a stable configuration condition given by $-1 \leqslant g \leqslant 1$ The round trip magnification coefficient $M$ may be calculated as the eigenvalues of the cavity matrix $A B C D$ Using the self consistency condition that the wavefront radius of the spherical wave is unchanged after one round trip, the two eigenvalues are given by

$M_{ \pm}=g \pm\left(g^{2}-1\right)^{1 / 2}$,

where the + and - signs denote the eigenvalues for the positive and negative branch resonators The round trip loss coefficient $L$ can be obtained by a simple geometric consideration as the ratio between the initial wave front area and the wave front area after one round trip It is given by

$L=-\ln \left(1 / M^{2}\right)$

The focusing cell contacted with one of the mirrors, considering a gaussian mode approxımation, acts like a spherical positive lens of variable power which depends on the field intensity in the cavity As we are lookıng for the dependence of the parameter $g$ on the field intensity, we will obtain an expression that relates the cavity configuration with the intensity The expression of the self focusing length $z_{\mathrm{f}}$ in a cell of width $d$, assuming the aberrationless approximation $[6,7]$, is $z_{\mathrm{f}}=a^{2} / 2 n_{2} d E^{2}$ where $a$ is the spot size in the cell, $n_{2}$ is the second order refractive index and $E$ is the field amplitude in the cavity Using this expression, the effective radius of curvature of the mirror contacted with the focusing cell is given by

$1 / R_{2}=1 / R+1 / z_{\mathrm{f}}$,

where $R$ is the "fixed" mirror radius

Replacing in eq (4) the self focusing length $z_{\mathrm{f}}$ we obtain

$1 / R_{2}=1 / R+\gamma \alpha I$,

where $\alpha=2 d / a^{2}$ and $\gamma I=n_{2} E^{2}$ The coefficient $\alpha$ takes into account the geometry of the resonator and the focusing cell, and $\gamma$ takes into consideration the nonlinear characteristics of the product Finally, replacing the effective radius of curvature $R_{2}$ in the definition of $g$, we obtain

$g=g_{0}-\beta I$

where $\quad g_{0}=1-2 L_{\mathrm{c}} / R-2 L_{\mathrm{c}} / R_{1}+2 L_{\mathrm{c}}{ }^{2} / R R_{1} \quad$ and $\beta=2 L_{\mathrm{c}} \gamma \alpha\left(1-L_{\mathrm{c}} / R_{\mathrm{l}}\right) \mathrm{Eq}(6)$ gives the intensity dependence of the parameter $g$ This equation describes the evolution of the cavity configuration when the intensity in the cavity changes and allows the evaluation of the loss coefficient as a function of the intensity The temporal evolution of the cavity intensity is thus obtained by placing the adequate loss coefficient in the evolution rate equations The nonlinear parameter $\beta$ completely defines the characteristics of the focusing cell and is the crucial design parameter The fact that permits the use of the self focusing effect as a passive gain modulator is that the cavity loss coefficient $L$ has a strong variation in a small range of intensities This behavior produces the 
Q-switch in the cavity as with saturable absorbers Nevertheless, in this modulation technique, there is a qualitative difference At higher intensities, the cavity configuration becomes unstable (for $g<-1$ ) and in this way, a limitation in the power obtainable exists The existence of a high loss branch for high intensities is characteristic of this modulation technique and was confirmed by our experimental results Another qualitative difference compared with saturable absorbers is the variation rate in the loss coefficient $L$ with the photon density $\phi(\mathrm{d} L / \mathrm{d} \phi)$ For a saturable absorber, $\mathrm{d} L / \mathrm{d} \phi$ decreases slowly to the saturated value of the $L$ coefficient as the intensity increases For the self focusing system, $\mathrm{d} L / \mathrm{d} \phi$ continuously increases as the intensity increases This fact ensures the fulfillment of the second threshold condition (the Q-switch condition) $[8,9]$ at higher intensities [2] In order to evaluate the temporal behavior of the photon density $\phi$ in the cavity, a numerical simulation using rate equations for $\phi$ and the amplification coefficient $A$ was performed The equations used in the simulation are

$\partial \phi / \partial t=(A-L-\Gamma) \phi / T_{\text {cav }}$,

$\partial A / \partial t=\left(A-A_{0}\right) / T_{\mathrm{a}}-\phi \sigma_{\mathrm{a}} A+P$,

where $A_{0}$ is the unsaturated amplification coefficient, $T_{\text {cav }}$ is the round trip time, $\Gamma$ is the linear loss coefficient (external coupling, scattering, absorption, etc ), $P$ is the pumping rate and $T_{\mathrm{a}}$ and $\sigma_{\mathrm{a}}$ are the decay time and the amplification cross section of the laser medium respectively The diffraction loss coefficient $L$ as defined in eq (3), can be evaluated as a function of time with eqs (7) which defines the temporal evolution of the cavity intensity The critical initial parameters that define the behavior of the system are $g_{0}$ (initial cavity parameter) and $\beta$ (nonlinear parameter) In fig 2, numerical simulations of the photon density $\phi$ versus $k$ (number of round trips) are shown The numerical simulations were performed using parameters corresponding to a Nd glass amplifier This is one of the laser media suitable for this modulation technique [2] Fig 2a shows the curves obtained for different values of $\beta$ It is clear that the photon flux evolution $\phi(k)$ has two different behaviors in the first case given by the curve 1 , the nonlinear parameter $\beta$ is small, and the loss saturation does not occur before the gain satu-
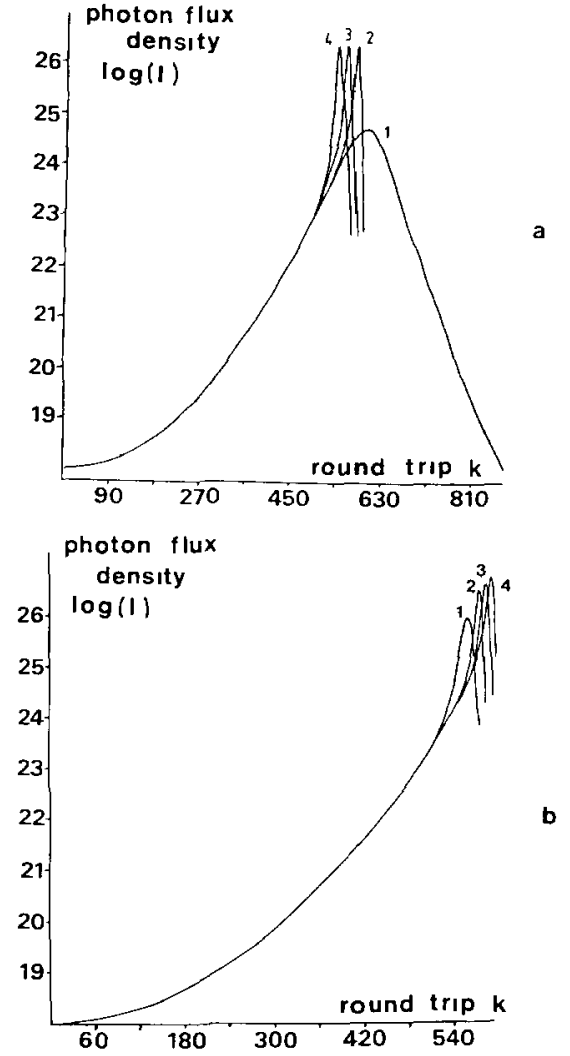

Fig 2 (a) Numerical simulation of the photon flux density $\phi$ versus the round trip number $k$ for different values of the nonlınear parameter $\beta \quad 1 \beta=10^{-9} \mathrm{~cm}^{2} / \mathrm{W} \quad 2 \beta=4 \times 10^{-9} \mathrm{~cm}^{2} / \mathrm{W} \quad 3$ $\beta=2 \times 10^{-8} \mathrm{~cm}^{2} / \mathrm{W} 4 \beta=5 \times 10^{-8} \mathrm{~cm}^{2} / \mathrm{W}$ In all cases $g_{0}=1016$ (b) For different values of the initial cavity parameter $g_{0} 1$ $g_{0}=100522 g_{0}=10253 \quad g_{0}=104 \quad 4 \quad g_{0}=1055$ In all cases $\beta=10^{-8} \mathrm{~cm}^{2} / \mathrm{W}$

ration We use here the term "loss saturation" as a parallelism with the saturable absorber method, but in this case, the loss saturation corresponds to the moment in which a stable cavity configuration is achieved In this case, the system cannot reach a stable configuration, and the result is a wide pulse of low power The other three curves represent the second case where the system reaches the second threshold condition, and a high power pulse takes place The puise width predicted (in the Q-switch regime) varies between $66 \mathrm{~ns}$ for $\beta=4 \times 10^{-9} \mathrm{~cm}^{2} / \mathrm{W}$ to $55 \mathrm{~ns}$ for $\beta=5 \times 10^{-8} \mathrm{~cm}^{2} / \mathrm{W}$ The different values of the parameter $\beta$ only slightly change the moment when the loss saturation occurs and the same high power pulse is obtained In the second case, the power pre- 
dicted by the numerical simulation is about $500 \mathrm{~kW}$

Fig $2 \mathrm{~b}$ shows the numerical simulations changing the initial cavity parameter $g_{0}$ In this case, we obtained pulses with decreasing power as $g_{0}$ approaches 1 (stable configuration) This fact enhances the influence of the nonlinear losses $(L)$ instead of the linear losses $(\Gamma)$ For this numerical simulations, there is a clear correspondence between the initial cavity configuration and the final pulsewidth For a defined unstable configuration ( $g_{0}=1055$ and $L=066$ ), the predicted pulsewidth is about 50 ns In a nearly stable configuration $\left(g_{0}=1005\right.$ and $L=02$ ), the simulation predicts a pulsewidth of 96 ns For this sequence of results, the parameter $\beta$ was chosen in order to assure the second threshold condition fulfillment We also made simulations without considering the focusing cell With this in mind, curves with a peak power 20 times lower than curves $2-4$ of fig 2a and a mean width of 380 to $400 \mathrm{~ns} \mathrm{sim-}$ lar to the curve 1 of fig $2 a$ were obtained

\section{Experimental results}

The experimental set-up is shown in fig 1 The laser amplifier was a KIGRE Q-88 phosphate Nd glass rod The cavity was composed by two maximum reflectıng mirrors with radius of curvatures of 2 and $-1 \mathrm{~m}$ The cavity length $L_{\mathrm{c}}$ was fixed in order to obtain an unstable configuration in the positive branch, and it could be changed in order to obtain different initial loss coefficients A $4 \mathrm{~cm}$ width focusing cell was contacted with the divergent mirror The cell was filled with phenyl salicylate (SALOL) [10] We also could control the SALOL temperature to within $05^{\circ} \mathrm{C}$ by an electrical heater The SALOL temperature was tested by a calibrated thermistor immersed in the product The output coupling (and then the linear losses $\Gamma$ ) could be continuously changed with a quarter wave plate and a polarizing cube The system was tested for different values of the initial cavity parameter $g_{0}$ and the nonlinear index $n_{2}$ by changing the cavity length and SALOL temperature respectively $[11,12] \quad F_{1 g} 3$ shows a sequence of oscilloscope traces of typical Q-switch pulses obtained with this modulation method For a fixed value of the $g_{0}$ parameter, we changed the SALOL temperature between $25^{\circ} \mathrm{C}$ (supercooled)
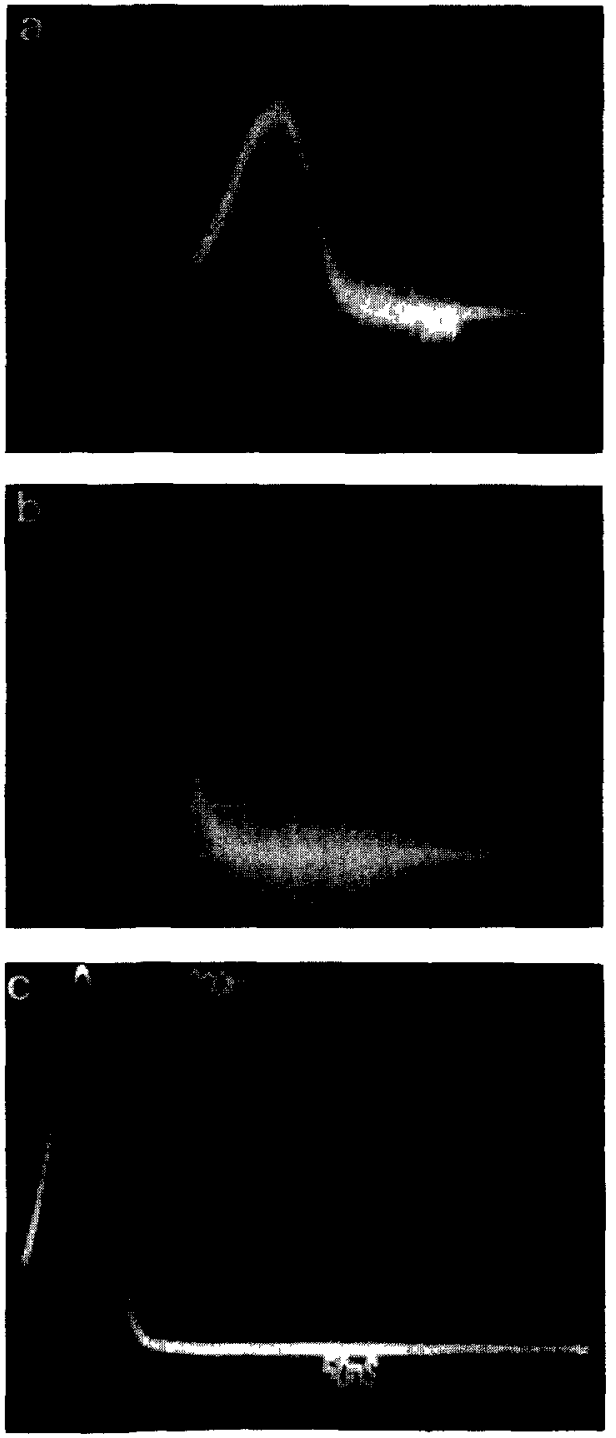

Fig 3 Sequence of experimental results changing the temperature of the focusing medium $1 T=25^{\circ} \mathrm{C}\left(\beta=10^{-8} \mathrm{~cm}^{2} / \mathrm{W}\right) 2$ $T=50^{\circ} \mathrm{C}\left(\beta=7 \times 10^{-9} \mathrm{~cm}^{2} / \mathrm{W}\right) \quad 3 \quad T=60^{\circ} \mathrm{C} \quad\left(\beta=54 \times 10^{\circ}\right.$ $\left.\mathrm{cm}^{2} / \mathrm{W}\right)$ In all cases $g_{0}$ is fixed at $1016\left(L_{\mathrm{c}}=985 \mathrm{~cm}\right)$

and $60^{\circ} \mathrm{C}$ This corresponds to a variation of the nonlınear index $n_{2}$ from $46 \times 10^{-12}$ (esu) to $25 \times 10^{-12}$ (esu) [12] This change means a variation in the nonlinear parameter $\beta$ between $\beta=10^{-8}$ $\mathrm{cm}^{2} / \mathrm{W}$ for $T=25^{\circ} \mathrm{C}$ and $\beta=55 \times 10^{-9} \mathrm{~cm}^{2} / \mathrm{W}$ for $T=60^{\circ} \mathrm{C}$ In this test, we obtanned almost the same pulsewidths with a fwhm of 50 ns to 75 ns and with a power of about $400 \mathrm{~kW}$ Both the pulsewidth and 

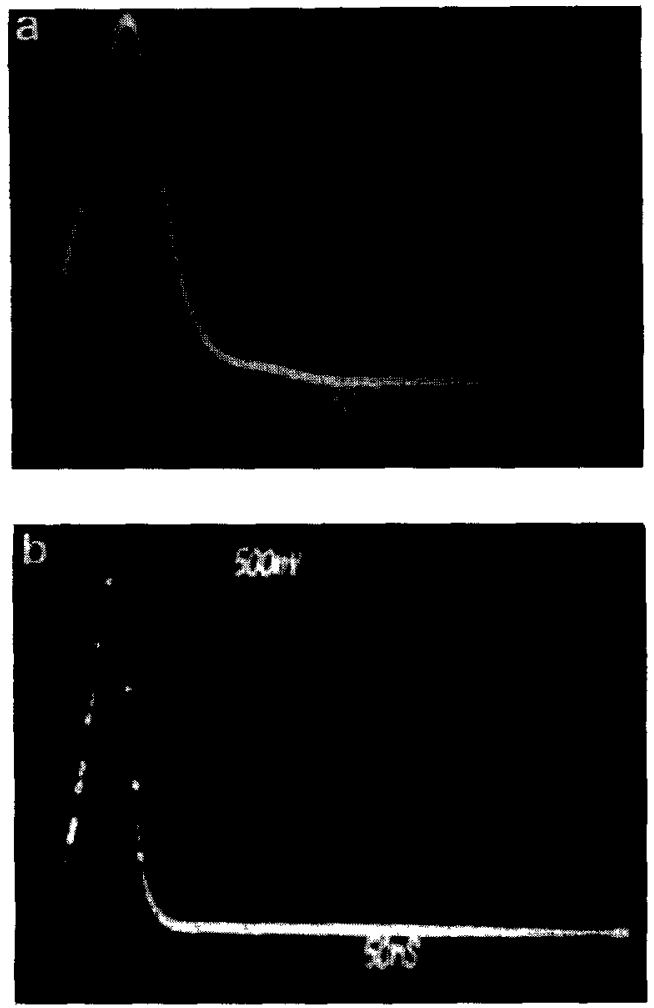

Fig 4 Experimental results changing the cavity length $1 L_{c}=985$ $\mathrm{cm}\left(g_{0}=1016\right) 2 L_{c}=92 \mathrm{~cm}\left(g_{0}=107\right)$ In both cases the temperature is fixed at $50^{\circ} \mathrm{C}\left(\beta=7 \times 10^{-9} \mathrm{~cm}^{2} / \mathrm{W}\right)$

the power obtained were very repetıtive for each temperature tested

Fig 4 shows the pulses obtained changing the inıtial cavity parameter $g_{0}$ for a fixed value of the SALOL temperature We tested two cavities lengths of $L_{\mathrm{c}}=985 \mathrm{~cm} \quad\left(g_{0}=1016\right)$ and $L_{\mathrm{c}}=92 \mathrm{~cm}$ $\left(g_{0}=107\right)$ where we fixed the SALOL temperature at $50^{\circ} \mathrm{C}\left(\beta=7 \times 10^{-9} \mathrm{~cm}^{2} / \mathrm{W}\right)$ A narrower pulse was obtained in the case of a more unstable initial configuration ( $\left.g_{0}=107\right)$ This fact was predicted by the numerical results presented in the previous section The pulsewidths varied between $40 \mathrm{~ns}$ and $75 \mathrm{~ns}$ and were in good agreement with the numerical results We also confirmed in this case a repetitive average power of 400 to $500 \mathrm{~kW}$ In all cases, the external coupling was fixed at the same value equivalent to a linear loss of $\Gamma=022$

Without the focusing cell, a multiple pulse emission was obtained with a peak intensity 20 to 25 times

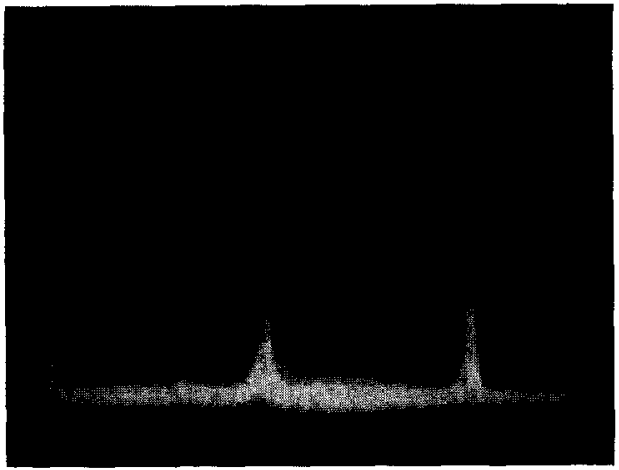

Fig 5 Multiple pulse emission obtained without the focusing cell

smaller and a pulsewidth of about $400 \mathrm{~ns}$ This is shown in fig 5

When placing the focusing cell into an initial stable cavity configuration, the emission has a strong modulation with short pulses like in a mode-locking regime The pulse separation was the round trip time and the total pulsewidth of the whole train varied between $600 \mathrm{~ns}$ and $800 \mathrm{~ns}$ In the fig 6a a typical pulse in this regime is shown When the focusing cell was eliminated, the short pulse emission completely disappeared and we obtained a wide pulse like the one in fig $6 \mathrm{~b}$ This modulation could be explained if we took into account the average power into the cavity was enough to produce an induced focal length in the cell to make the cavity an unstable resonator When the intensity decreases because of the high loss, a stable configuration was again achieved, and a new pulse could buld up Nevertheless, this effect has a qualitative difference with the saturable absorber passive mode-locking At high intensities, the self focusing effect introduced a high loss that makes the intensity decrease $W_{1}$ th the saturable absorber, the bleaching process makes a simultaneous Q-switch that in our system was not observed

As a last remark, we observed a filament free emission in all the experimental conditions performed We also used the same focusing product for several months without changes occurring in its nonlinear characteristics

\section{Conclusions}

A novel passive technique has been presented in detail by a simple theoretical model and by a com- 

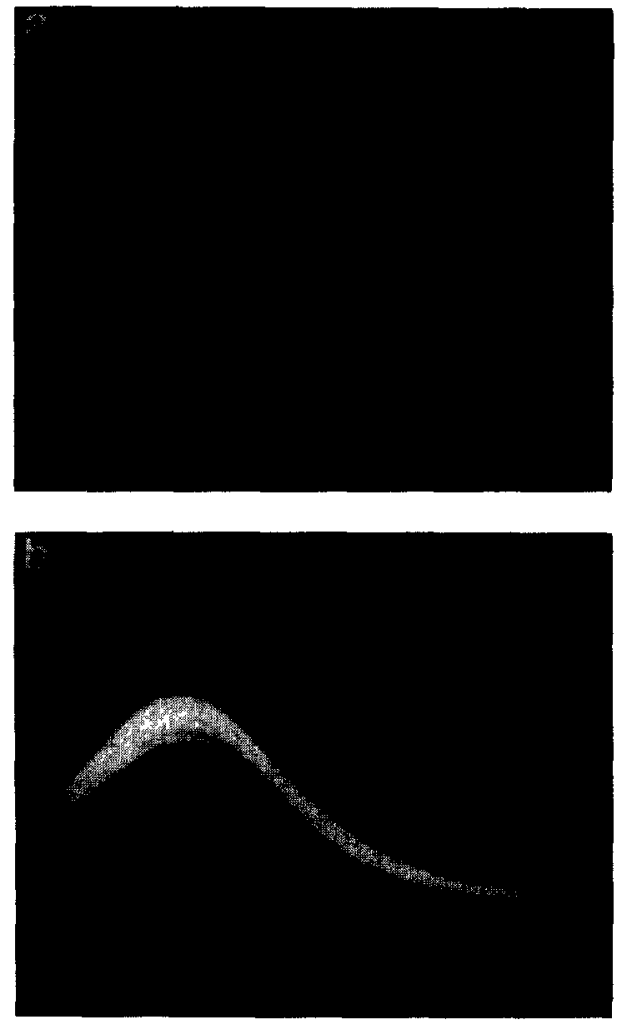

Fig 6 (a) Short pulse train obtained placing the focusing cell in a stable configuration cavity (b) Pulse obtained when the focusing cell is elımınated

plete series of experımental results which are in good agreement with the numerical simulations performed This passive modulation method provides some advantages As the self focusing effect depends on the field intensity, this modulation technique is not restricted in wavelength The radiation builds up from an unstable resonator and the generation of smooth modes are favored, which provides a good mode quality The high loss coefficient at high intensities avoids the filament emission This fact was experımentally verified The nonlinear material used in the focusing cell is more stable than saturable absorbers and can be used without notable changes in the laser emission over long periods of time It is possible to control the nonlinear index $n_{2}$ and the recovery time of the product by changing the tem- perature [12] We verify that the change in the recovery time does not affect the short pulse generation For all temperatures tested, the recovery time $\tau$ was smaller than the round trip time Nevertheless, we also performed numerical simulations taken into account the "inertia" of the focusing product for the different temperatures In all cases we obtain the same short pulse as in the former case when an inertialess nonlinear medium was considered

Placing the focusing cell into a stable cavity, we obtained a short pulse emission like in a mode locking regime with a repetitive behavior The study of this effect will be the subject of further research

A drawback of this technique compared to saturable absorbers is its higher sensitivity to misalignments A very stable table and mirror positioners should be used in order to obtain a repetitive emission

An energy limitation arising because an unstable cavity configuration occurs at high intensities was also predicted and confirmed experimentally We also confirmed the pulsewidth and the peak power predicted by the theoretical model in the different conditions tested

\section{References}

[1] M C Marconı, O E Martınez and F P D1odatı, Optıcs Lett $10(1985) 402$

[2] M C Marconı, O E Martınez and F P Diodatı, J Opt Soc Am 3 (1986) 12

[3] L W Casperson, IEEE J Quant Electron QE-10 (1974) 629

[4] A E Siegman, IEEE J Quant Electron QE-12 (1976) 35

[5] A E Siegman, Proc IEEE 53 (1965) 227

[6] P D McWane, Nature 211 (1966) 1081

[7] S A Akhamanov, R V Khokhlov and A Sukhorukov, in Laser handbook eds F T Arecch1 and O E Schulz-Dubors (North Holland Publıshıng Co, Amsterdam, 1972) p 1173

[8] G H C New and T B O'Hare, Phys Lett A68 (1978) 28

[9] G H C New, Proc IEEE 67 (1979) 380

[10] The SALOL used in this experiment was provided by the Chemical Division of Rhone-Poulenc Inc

[11] P P Ho and R R Alfano, J Chem Phys 67 (1977) 1004

[12] P P Ho and R R Alfano, Phys Rev A 20 (1979) 2170 